\title{
Novel Properties for Total Strong - Weak Domination Over Bipolar Intuitionistic Fuzzy Graphs
}

\author{
As'ad Mahmoud As'ad Alnaser \\ Department of Applied Science \\ Ajloun College, Al-Balqa Applied University \\ Jordan
}

\begin{abstract}
Through this research study, we introduced and discussed total strong (weak) domination concept of bipolar intuitionistic fuzzy graphs and in define strong domination bipolar intuitionistic fuzzy graph also strong domination. Theorems, examples and some properties of these concept are discussed.
\end{abstract}

Keywords-Fuzzy sets; bipolar intuitionistic fuzzy sets; strong (weak) bipolar intuitionistic fuzzy sets; total strong (weak) bipolar intuitionistic number

\section{INTRODUCTION}

The theory of fuzzy sets was introduced by Zadeh [1]. On this notion the researchers emphasized their applications in different areas such as electrical engineering economics, Computer Science, social networks, system analysis and mathematics, many researchers using this concept to generalized and study some topics [2-8]. Atanassov [9] generalized the idea of fuzzy set and gave new Concept which intuitionistic fuzzy sets. Many researchers have benefited from this new Concept in developing many old Concepts in many fields of Science [10-13]. Zhang [14] initiated a bipolar fuzzy set concept as a development to the fuzzy set theory, since a set of bipolar fuzzy is an extension of fuzzy set of Zadeh's whose membership degree range is $[-1,1]$. Also, many researchers have used this notion to study many properties [15-18]. E zhilmaran and sankar [19 - 20] have introduced bipolar intuitionistic fuzzy set and studied it on graph theory, A.Alnaser et.al in 2020 [21] used this concept to graph theory also. The concept of bipolar intuitionistic fuzzy set is considered a new and important concept as it has entered many sciences such as networks and engineering, mathematics, control systems, medicine and other sciences. In our future study we will use this concept to develop some of the results reached in many research papers such as [22-23].

In this paper, we will introduce and discuss total strong (weak) domination concept of bipolar intuitionistic fuzzy graphs and define strong domination bipolar intuitionistic fuzzy graph \& strong domination. Theorems, examples and some properties of these concepts will also be discussed.

\section{PRELIMINARIES}

Definition 2.1: [1] Let $\mathrm{G}$ be a set, a fuzzy set $\delta$ on $G$ just function $\delta: G \rightarrow[0,1]$.

Definition 2.2: [3] A fuzzy set $\delta$ is said to be fuzzy relation on $G$ if the map $\mathrm{\gamma}: G \times G \rightarrow[0,1]$ satisfy $\mathrm{\gamma}(a, d) \leq$ $\min \{\delta(a), \delta(d j)\}$ for all $a, d \in G$. A fuzzy relation is symmetric if $\mathrm{y}(a, d)=\mathrm{\gamma}(d, a)$ for all $a, d \in G$.

Definition 2.3: [14] If $G \neq \varphi$. A bipolar fuzzy set $\lambda$ of $G$ is object with form $\varphi=\left\{\left(i, \lambda^{+}(i), \lambda^{-}(i) ; i \in G\right)\right\}$ such that $\lambda^{+}: G \rightarrow[0,1]$ and $\lambda^{-}: G \rightarrow[-1,0]$ are mappings.

Definition 2.4: [9] If $G$ is anon empty set. An intuitionistic fuzzy set $\mathfrak{I}=\{(k ; \mu(k), \lambda(k) ; k \in G)\}$ such that $\mu: G \rightarrow$ $[0,1]$ and $\lambda: G \rightarrow[0,1]$ are mapping such that $0 \leq$ $\mu(k)+\lambda(k) \leq 1$.

Definition 2.5: [24] An ordered pair $G^{*}=(V, E)$ is graph such that $V$ the vertices set in $G^{*} \& E$ the edge set in $G^{*}$.

Remark 2.6: [24] 1) If $c$ and $e$ are two vertices in $G^{*}$ then its called adjacent of $G^{*}$ when $(c, e)$ is edge of $G^{*}$.

2) An undirected graph which has at most one edge between any two different vertices no loops called simple graph.

Definition 2.7: [24] A sub graph of $G^{*}$ is a graph $S=$ ( $W, F$ ) such that $W \leq V$ and $F \leq E$.

Definition 2.8: [24] $\left(G^{*}\right)^{C}$ is complementary graph of a simple graph with the same vertices of $G^{*}$.

Remark 2.9: [24] Two vertices are adjacent in $\left(G^{*}\right)^{C}$ iff they are not adjacent in $G^{*}$.

\section{Mean Results}

Definition 3.1: [19] If $G \neq \varphi$. A bipolar intuitionistic fuzzy sets $\mathfrak{I}=\left\{(e) \mu^{+}(e), \mu^{-}(e), \lambda^{+}(e), \lambda^{-}(e) ; e \in G\right\}$ such that $\mu^{+}: G \rightarrow[0,1], \mu^{-}: G \rightarrow[-1,0], \lambda^{+}: G \rightarrow[0,1]$, $\lambda^{-}: G \rightarrow[-1,0]$. Are mapping, where $0 \leq \mu^{+}(e)+$ $\lambda^{+}(e) \leq 1,-1 \leq \mu^{-}(e)+\lambda^{-}(e) \leq 0$.

Using the degree of positive membership $\mu^{+}(i)$ to represent the degree of satisfaction of " $e$ " to the corresponding of property of a bipolar intuitionistic fuzzy sets $\mathfrak{J}$ also the negative degree of membership $\mu^{-}(e)$ for represent the satisfaction degree of " $e$ " for any implicit counter property corresponding for a bipolar intuitionistic fuzzy sets. By the same cases, we use the degree of positive none membership $\lambda^{+}(e)$ for represent the satisfaction degree of " $e$ " to the property corresponding for a bipolar intuitionistic fuzzy sets also, the degree of negative non membership $\lambda^{-}(e)$ for represent the satisfaction degree " $e$ " to some implicit counter property corresponding for a bipolar intuitionistic fuzzy sets. 
If $\mu^{+}(e) \neq 0, \mu^{-}(e)=0$ and $\lambda^{+}(e)=0, \lambda^{-}(e)=0$ the situation that " $e$ " regarded as have only a positive membership property in bipolar intuitionistic fuzzy sets. While if $\mu^{+}(e)=0, \mu^{-}(e) \neq 0$ also $\lambda^{+}(e)=0, \lambda^{-}(e)=0$, then it's the situation that " $e$ " regarded as have a negative member ship property. While if $\mu^{+}(e)=0, \mu^{-}(e)=0$ also $\lambda^{+}(e) \neq$ $0, \lambda^{-}(e)=0$, it's the situation that " $e$ regarded as have only a positive non member - ship property. If $\mu^{+}(e)=0, \mu^{-}(e)=$ 0 also $\lambda^{+}(e)=0, \lambda^{-}(e) \neq 0$ it's a situation that " $e$ " regarded as have only the negative non member - ship property. It is possible for an element $e$ to be such that $\mu^{+}(e) \neq 0, \mu^{-}(e) \neq$ 0 also $\lambda^{+}(e) \neq 0, \lambda^{-}(e) \neq 0$ when a member - ship and non member - ship function of a property overlaps with its counter properties over some one portion of " $e$ ".

Definition 3.2: [19] If $G$ is anon empty set. Then the mapping $\quad \mathfrak{I}=\left(\mu_{\mathfrak{\Im}}^{+}, \mu_{\mathfrak{I}}, \lambda_{\mathfrak{\Im}}^{+}, \lambda_{\mathfrak{J}}\right): G \times G \rightarrow([0,1] \times$ $[-1,0] \times[0,1] \times[-1,0])$ is a bipolar intuitionistic fuzzy relation on $G$, where $\mu_{\mathfrak{J}}^{+}(i, j) \in[0,1], \mu_{\mathfrak{I}}(i, j) \in$ $[-1,0], \lambda_{\mathfrak{J}}^{+}(i, j) \in[0,1]$ and $\lambda_{\mathfrak{J}}^{-}(i, j) \in[-1,0]$.

Definition

3.3:

$\mathfrak{\Im}_{1}=\left(\mu_{\mathfrak{\Im} 1}^{+}(e), \mu_{\mathfrak{I}_{1}}(e), \lambda_{\mathfrak{\Im} 1}^{+}(e), \lambda_{\mathfrak{I}_{1}}(e)\right.$

$\left(\mu_{\mathfrak{I} 2}^{+}(e), \mu_{\mathfrak{\Im} 2}(e), \lambda_{\mathfrak{\Im} 1}^{+}(e), \lambda_{\mathfrak{\Im}_{2}}(e)\right.$ be two bipolar intuitionistic fuzzy sets on $G . \Im_{1}$ is a bipolar intuitionistic fuzzy relation for $\mathfrak{I}_{2}$ if

1) $\mu_{\mathfrak{I} 1}^{+}(e, f) \leq \min \left\{\mu_{\mathfrak{I} 2}^{+}(e), \mu_{\mathfrak{I} 2}^{+}(f)\right\}$

2) $\mu_{\Im}^{-}(e, f) \geq \max \left\{\mu_{\mathfrak{J} 2}^{-}(e), \mu_{\mathfrak{S} 2}^{-}(f)\right\}$

3) $\lambda_{\mathfrak{I} 1}^{+}(e, f) \geq \max \left\{\lambda_{\mathfrak{I} 2}^{+}(e), \lambda_{\mathfrak{I} 2}^{+}(f)\right\}$

4) $\lambda_{\mathfrak{J} 1}(e, f) \leq \min \left\{\lambda_{\mathfrak{J} 2}^{-}(e), \lambda_{\mathfrak{J} 2}^{-}(f)\right\}$

5) $\forall e, f \in G$.

Remark 3.4: A bipolar intuitionistic fuzzy relation for $\mathfrak{I}_{1}$ on $G$ is said to be symmetric when $\mu_{\mathfrak{I} 1}^{+}(e, f)=\mu_{\mathfrak{I} 1}^{+}(f, e)$, $\mu_{\overline{\mathfrak{I}} 1}(e, f)=\mu_{\mathfrak{I}_{1}}(f, e)$

$\lambda_{\mathfrak{I} 1}^{+}(e, f)=\lambda \stackrel{+}{\mathfrak{1} 1}(f, e), \lambda \overline{\mathfrak{I}}_{1}(e, f)=\lambda \overline{\mathfrak{I}}_{1}(f, e)$.

$\forall e, f \in G$.

Definition 3.5: [19] A bipolar intuitionistic fuzzy graph of $G^{*}=(V, E)$ that is a pair $G=(X, Y)$ such that $\mathfrak{I}_{1}=$ $\left(\mu_{\mathfrak{I} 1}^{+}, \mu_{\mathfrak{I} 1}^{-}, \lambda_{\mathfrak{I} 1}^{+}, \lambda_{\overline{\mathfrak{I}}_{1}}^{-}\right)$is a bipolar intuitionistic fuzzy sets in $V$ and $\mathfrak{I}_{2}=\left(\mu_{\mathfrak{J}_{2}}^{+}, \mu_{\mathfrak{\Im}_{2}}, \lambda_{\mathfrak{J}_{2}}^{+}, \lambda_{\mathfrak{\Im}_{2}}\right)$ is a bipolar intuitionistic fuzzy set of $V \times V$ such that.

1) $\mu_{\mathfrak{I} 2}^{+}(e, f) \leq \min \left\{\mu_{\mathfrak{I} 1}^{+}(e), \mu_{\mathfrak{I} 1}^{+}(f)\right\}, \forall$ ef $\in V \times V$ V

2) $\mu_{\overline{\mathfrak{I}}}(e, f) \geq \max \left\{\mu_{\overline{\mathfrak{I}}}(e), \mu_{\mathfrak{I}_{1}}^{-}(f)\right\}, \forall$ ef $\in V \times$

3) $\lambda \underset{\Im 3}{+}(e, f) \geq \max \left\{\lambda_{\mathfrak{I} 1}^{+}(e), \lambda_{\mathfrak{J 1}}^{+}(f)\right\}, \forall$ ef $\in$ $V \times V$

$V \times V$

4) $\lambda \overline{\mathfrak{J}_{2}}(e, f) \leq \min \left\{\lambda \overline{\mathfrak{J}_{1}}(e), \lambda_{\mathfrak{I}_{1}}(f)\right\}, \forall$ ef $\in$

5) $\mu_{\mathfrak{I} 2}^{+}(e, f)=\mu_{\mathfrak{I} 2}^{-}(e, f)=0 \forall$ ef $\in V \times V-E$

6) $\lambda_{\mathfrak{I} 2}^{+}(e, f)=\lambda_{\mathfrak{I} 2}^{-}(e, f)=0 \forall$ ef $\in V \times V-E$

Through this article, $G^{*}$ and $G$ is representing a crisp to a graph and bipolar intuitionistic fuzzy graph respectively.

Definition 3.6: Let $F=\left(\mathfrak{I}_{1}, \mathfrak{I}_{2}\right)$ be a bipolar intuitionistic fuzzy graphs, where

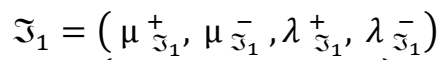
and $\mathfrak{I}_{2}=\left(\mu_{\mathfrak{I}_{2}}^{+}, \mu_{\mathfrak{I}_{2}}, \lambda_{\mathfrak{J}_{2}}^{+}, \lambda_{\mathfrak{J}_{2}}\right)$ are two bipolar intuitionistic fuzzy set on a non empty set $V$ and $E \leq V \times V$ respectively.

The positive degree of a vertex $\mu_{\mathfrak{\Im}_{1}}^{+}, \lambda{\stackrel{\mathfrak{\Im}}{\mathfrak{I}_{1}}}^{+} \in G$ is $D^{+}\left(\mu_{\mathfrak{J}_{1}}^{+}(e), \lambda_{\mathfrak{J}_{1}}^{+}(e)\right)=\sum_{e f \in E} \mu_{\mathfrak{J}_{2}}^{+}(e f)+\sum_{e f \in E} \lambda_{\mathfrak{I}_{2}}^{+}(e f)$

Similarly, the negative degree of a vertex $\mu_{\overline{\mathfrak{I}}_{1}}, \lambda \overline{\overline{\mathfrak{I}}_{1}} \in G$ is $D^{-}=\left(\mu_{\mathfrak{\Im}_{1}}(e), \lambda_{\mathfrak{I}_{1}}(e)\right)=\sum_{e f \in E} \mu_{\widetilde{\mathfrak{I}}_{2}}(e f)+\sum_{e f \in E} \lambda_{\mathfrak{I}_{2}}(e f)$.

The degree of the vertex is $D(\mu, \lambda)=$ $\left(D^{+}(\mu, \lambda), D^{-}(\mu, \lambda)\right.$.

Definition 3.7: If $G=\left(\mathfrak{I}_{1}, \mathfrak{I}_{2}\right)$ is bipolar intuitionistic fuzzy graphs. Then the bipolar intuitionistic fuzzy graph $G$ order given by $O(G)=$

$\left(\sum_{e \in V} \mu_{\mathfrak{I}_{1}}^{+}(e), \sum_{f \in V} \lambda_{\mathfrak{I}_{1}}^{+}(f), \sum_{e \in V} \mu_{\mathfrak{J}_{1}}(e), \sum_{e \in V} \lambda_{\mathfrak{\Im}_{1}}^{-}(f),\right)$.

The size of a bipolar intuitionistic fuzzy graph $G$ is $S(G)=$

$\left(\sum_{i j \in V} \mu_{\mathfrak{J}_{1}}^{+}(i j), \sum_{i j \in V} \mu_{\widetilde{J}_{1}}^{-}(i j), \sum_{i j \in V} \lambda_{\mathfrak{J}_{1}}^{+}(i j), \sum_{i j \in V} \lambda_{\widetilde{J}_{1}}^{-}(i j)\right)$

Definition 3.8: if $G=\left(\mathfrak{I}_{1}, \mathfrak{I}_{2}\right)$ is a bipolar intuitionistic fuzzy graph, for each node in $G$ has the same closed degree of neighborhood, thus $G$ said to be totally bipolar intuitionistic fuzzy graphs. The closed degree of neighborhood of a node "e" defined as $\operatorname{Deg}(e)=\left(\operatorname{Deg}^{+}(e)+\operatorname{Deg}^{-}(e)\right)$, such that $\operatorname{Deg}^{+}(e)=\left(\operatorname{Deg}^{+}(e)+\mu_{\mathfrak{J 1}}^{+}(e), \operatorname{Deg} \lambda^{+}(e)+\lambda_{\mathfrak{I} 1}^{+}(e)\right)$

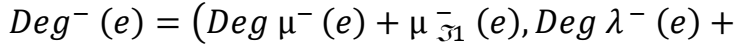

$$
\begin{aligned}
& \left.\lambda_{\mathfrak{I} 1}^{+}(e)\right) \text {. }
\end{aligned}
$$

Definition 3.9: A bipolar intuitionistic fuzzy graphs $G=\left(\mathfrak{I}_{1}, \mathfrak{I}_{2}\right)$ is called strong bipolar intuitionistic fuzzy graph, when $\mu_{\mathfrak{J}_{2}}^{+}(i j)=\min \left\{\mu_{\mathfrak{J}_{1}}^{+}(i), \mu_{\mathfrak{J}_{1}}^{+}(j)\right\}, \lambda_{\mathfrak{J}_{2}}^{+}(i j)=$ $\max \left\{\lambda_{\widetilde{J}_{1}}^{+}(i), \lambda_{\widetilde{J}_{1}}^{+}(j)\right\}$ $\mu_{{\widetilde{J_{2}}}_{2}}^{-}(i j)=\min \left\{\mu_{{\widetilde{J_{1}}}_{1}}^{-}(i), \mu_{{\widetilde{J_{1}}}_{1}}^{-}(j)\right\} \quad \lambda_{{\widetilde{J_{2}}}_{2}}^{-}(i j)=$ $\max \left\{\lambda \overline{\widetilde{J}_{1}}(i), \lambda \overline{\widetilde{J}_{1}}(j)\right\} \forall i j \in E$.

Definition 3.10: if $G=\left(\mathfrak{I}_{1}, \mathfrak{I}_{2}\right)$ is a bipolar intuitionistic fuzzy graph, if $I$ and $J$ are two vertices. Then $I$ is totally strong dominates $J$ ( $J$ totally weak dominates $I$ )

If

1) $\mu_{\mathfrak{J}_{2}}^{+}(e f)=\min \left\{\mu_{\widetilde{J}_{1}}^{+}(e), \mu_{\mathfrak{J}_{1}}^{+}(f)\right\}$, $\mu_{{\widetilde{J_{2}}}_{2}}^{-}(e f)=\min \left\{\mu_{{\mathfrak{J _ { 1 }}}_{1}}^{-}(e), \mu_{{\widetilde{J_{1}}}_{1}}(f)\right\}$ and

$\lambda_{\widetilde{J}_{2}}^{+}(e f)=\max \left\{\lambda_{\widetilde{J}_{1}}^{+}(e), \lambda{ }_{\widetilde{J}_{1}}^{+}(f)\right\}$ and $\lambda{\overline{\widetilde{J}_{2}}}^{-}(e f)=\max \left\{\lambda{\overline{\Im_{1}}}^{-}(e), \lambda{\overline{\Im_{1}}}^{-}(f)\right\} . \forall$ ef $\in E$

2) $D^{-}(I) \geq D^{-}(J)$ and

3) Every vertex in Gdominates I

Definition 3.11: If $G$ is a bipolar intuitionistic fuzzy graph; $\tau_{B}$ is called total strong ( weak ) dominating a bipolar intuitionistic set of $G$ if 


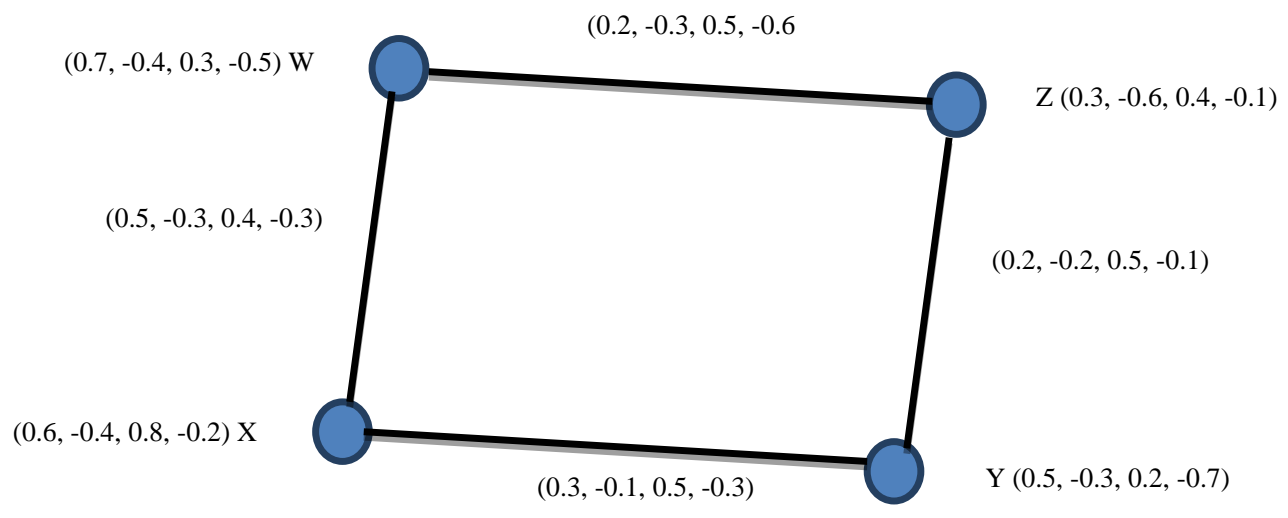

Fig. 1. Bipolar Intuitionistic Fuzzy Graph.

$V(G)$

1) $\mu(H, S) \geq \mu^{\infty}(H, S), \lambda(H, S) \geq \lambda^{\infty}(H, S) \forall H, S \in$

2) $\left.D^{-}(H) \geq D^{-}(S) \forall H \in \tau_{B}, S \in V-\tau_{B}\right)$

3) $\mu_{\mathfrak{J}_{2}}^{+}(e f)=\min \left\{\mu_{\mathfrak{J}_{1}}^{+}(e), \mu_{\widetilde{J}_{1}}^{+}(f)\right\}$

$\mu_{\widetilde{\mathfrak{J}_{2}}}(e f)=\min \left\{\mu_{\widetilde{\mathfrak{J}_{1}}}(e), \mu_{{\widetilde{J_{1}}}_{1}}(f)\right\}$ and

$\lambda \lambda_{\mathfrak{J}_{2}}^{+}(e f)=\max \left\{\lambda \underset{\widetilde{J}_{1}}{+}(e), \lambda \stackrel{+}{\mathfrak{J}_{1}}(f)\right\}$

$\lambda \overline{\widetilde{J}_{2}}(e f)=\max \left\{\lambda{\overline{\mathfrak{I}_{1}}}_{\widetilde{J}_{1}}(e), \lambda \overline{\widetilde{J}_{1}}(f)\right\} . \forall$ ef $\in E$

4) $\tau_{B}$ is the total dominating a bipolar intuitionistic set

Definition 3.12: $\tau_{B}$ of a fuzzy graphs $G$ is called minimal total strong (weak) dominating bipolar intuitionistic set of $G$, if there doesn't exist any total strong (weak) dominating bipolar intuitionistic set of $G$, whose cardinality is less than the cardinality $\tau_{B}$.

Definition 3.13: A total strong (weak) dominating bipolar intuitionistic set in $G$ is the fuzzy cardinality of minimum among all minimal total strong (weak) dominating bipolar intuitionistic set $G$.

Remark 3.14: The total strong (weak) domination bipolar intuitionistic number is represented by $\pi_{\tau_{B}}(G)$.

Example 3.15: If $G$ is a bipolar intuitionistic fuzzy graph given by the Fig. 1.

Total strong (weak) dominating bipolar intuitionistic set $\tau_{B}=\{x, y\}$

Total strong (weak) bipolar domination number $\pi_{\tau_{B}}\left(\mu \stackrel{+}{\mathfrak{J}_{1}}, \mu_{\mathfrak{J}_{1}}, \lambda \stackrel{+}{\mathfrak{I}_{1}}, \lambda \overline{\mathfrak{J}_{1}}\right)=(1.1,-0.7,1,-0.9)$

$\operatorname{Deg}\left(\mu_{x}^{+}, \mu_{x}^{-}, \lambda_{x}^{+}, \lambda_{x}^{-}\right)=(0.8,-0.4,0.9,-0.6)$

$\operatorname{Deg}\left(\mu_{y}^{+}, \mu_{y}^{-}, \lambda_{y}^{+}, \lambda_{y}^{-}\right)=(0.5,-0.3,1,-0.4)$

$\operatorname{Deg}\left(\mu_{z}^{+}, \mu_{z}^{-}, \lambda_{z}^{+}, \lambda_{z}^{-}\right)=(0.4,-0.5,1,-0.7)$

$\operatorname{Deg}\left(\mu_{w}^{+}, \mu_{w}^{-}, \lambda_{w}^{+}, \lambda_{w}^{-}\right)=(0.7,-0.6,0.9,-0.9)$

Order of bipolar intuitionistic fuzzy graph $O(G)=$ $(2.1,-1.7,1.7,-1.5)$

Size of bipolar intuitionistic fuzzy graph $S(G)=$ $(1.2,-0.9,1.9,-1.3)$
Theorem 3.16: If $G$ is bipolar intuitionistic fuzzy graph also if $\tau_{B}$ is minimal total strong (weak) dominating. Then for each $J \in \tau_{B}$, if one of following axioms hold

1) There is no vertex of $\tau_{B}$ strongly dominates $J$

2) $\exists J \in V-\tau_{B}$; is the only vertex in $\tau_{B}$ which strongly dominates $I$

3) $\tau_{B}$ is bipolar intuitionistic fuzzy graph with total dominating set.

Proof: Suppose that $\tau_{B}$ is a minimal total strong (weak) dominating set. Then for every $J \in \tau_{B}, \tau_{B}-\{J\}$ is not a total strong (weak) dominating set, then there exist $I \in V-\tau_{B}$, such that not strongly dominated by any vertex belong to $\tau_{B}-\{J\}$. Since $\tau_{B}$ is total strong dominating set. Thus $J$ is only vertex which strongly dominates $J$ and hence axiom 2 hold.

Now, suppose that $\tau_{B}$ be total strong (weak) dominating set also for each $J \in \tau_{B}$ thus one of the following two axioms holds

If $\tau_{B}$ not minimal, then $\exists J \in \tau_{B}, \tau_{B}-\{J\}$ a total strong dominating set also hence $J$ is strongly dominated by at least one vertex in $\tau_{B}-\{J\}$ and its contradiction by 1

If $\tau_{B}-\{J\}$ is a total strong (weak) dominating, then every vertex belong to $V-\tau_{B}$ is totally strong (weak) dominated by at least one vertex belong to $\tau_{B}-\{J\}$. The second condition does not holds. This $\tau_{B}$ a minimal total strong (weak) dominating set.

Corollary 3.17: All complete bipolar intuitionistic fuzzy graph also total strong (weak) domination in bipolar intuitionistic fuzzy graphs.

Proof: $G$ be complete bipolar intuitionistic fuzzy graphs. Thus every edge is total strong (weak) dominating set also every vertices are join together. Thus and obvious, $G$ be total strong (weak) dominating set.

Example 3.18: Given a bipolar intuitionistic fuzzy graphs $G$ given by Fig. 2 and 3 . 


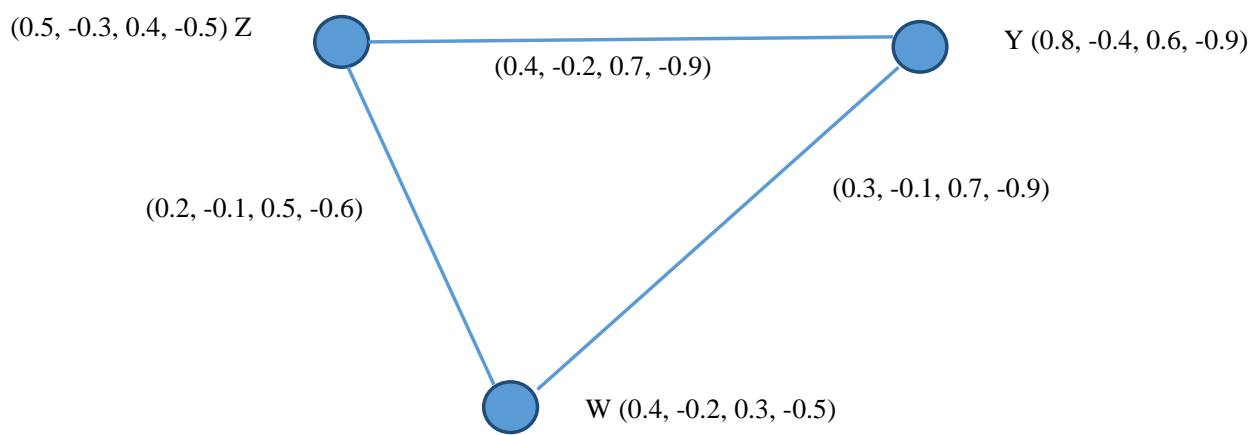

Fig. 2. Bipolar Intuitionistic Fuzzy Graph.

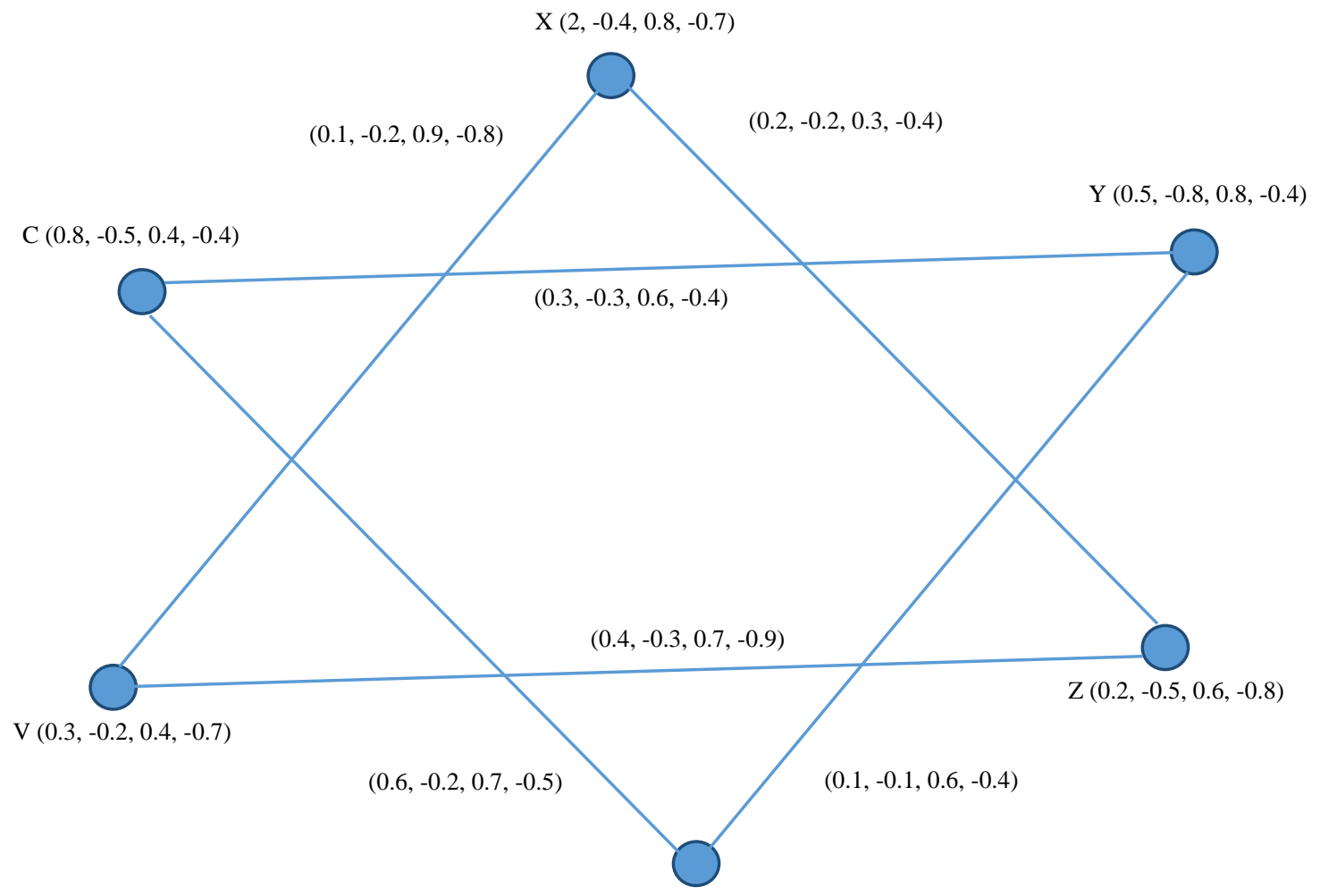

W $(0.7,-0.5,0.6,-0.4)$

Fig. 3. Bipolar Intuitionistic Fuzzy Graph.

$\tau_{B}-\{Z\}, \pi_{\tau_{B}}-\{G\}=(0.2,-0.1,0.5,-0.6)$

$\tau_{B}-\{W\}, \pi_{\tau_{B}}-\{G\}=(0.3,-0.9,0.4,-0.8)$

Definition 3.19: A bipolar intuitionistic fuzzy graphs $G$ said to be a semi - $\mathrm{\gamma}$ - strong bipolar intuitionistic fuzzy graph when $\mu_{\mathfrak{I} 2}^{+}(e, f)=\min \left\{\mu_{\mathfrak{I} 1}^{+}(e), \mu_{\mathfrak{I} 1}^{+}(f)\right\}$, and $\mu_{\mathfrak{I} 2}^{-}(e, f)=$ $\min \left\{\mu_{\tilde{J}_{1}}^{-}(e), \mu_{\mathfrak{I} 1}^{-}(f)\right\}$ for every $e$ and $f$.

Theorem 3.20: Let $G$ be a bipolar intuitionistic fuzzy graph on order $O(G)$ then

1) $\mathfrak{I}_{\text {sbif }}(G) \leq \mathfrak{I}_{\text {tsbif }}(G) \leq O(G)-\Delta_{n}(G) \leq$ $O(G)-\Delta_{e}(G)$
2) $\mathfrak{I}_{\text {sbif }}(G) \leq \mathfrak{I}_{t w b i f}(G) \leq O(G)-\delta_{n}(G) \leq$ $O(G)-\delta_{e}(G)$

Where $\mathfrak{I}_{\text {sbif }}, \mathfrak{I}_{\text {tsbif }}, \mathfrak{I}_{\text {twbif }}$ represent to strong bipolar intuitionistic domination, total strong bipolar intuitionistic domination and total strong (weak) bipolar intuitionistic domination respectively.

Proof: Hence $G$ every $\mathfrak{I}_{\text {sbifd }}-\operatorname{set}\left(\mathfrak{I}_{t w b i f d}-\right.$ set $)$ is a bipolar intuitionistic dominating set $\mathfrak{I}_{\text {sbif }}(G) \leq \mathfrak{I}_{\text {tsbif }}(G)$ and $\mathfrak{I}_{w b i f}(G) \leq \mathfrak{I}_{t w b i f}(G)$ if $i, j \in V$ and $D_{n}(i)=\Delta_{n}(G)$ and $D_{n}(i)=\delta_{n}(G)$ then $V-N(i)$ is a $\Im_{t s b i f d}-$ set but not minimal and $V-N(j)$ is $\mathfrak{I}_{\text {twbifd }}-$ set but not minimal 
hence $\mathfrak{I}_{t s b i f}(G) \leq|V-N(i)|_{\text {sbif }}$ this means that $\mid V-$

$\left.N(i)\right|_{\text {sbif }}=|V|-|N(u)|$

$\Rightarrow O(G)-D_{n}(u)$

$\Rightarrow O(G)-\Delta_{n}(G)$

$\Rightarrow \mathfrak{I}_{t s b i f}(G) \leq O(G)-\Delta_{n}(G)$ and $\mathfrak{I}_{t w b i f}(G) \leq$

$|V-N(i)|_{\text {wbif }}$

this means that $|V-N(j)|_{w b i f}=|V|-|N(j)|$

$\Rightarrow O(G)-D_{n}(j)$

$\Rightarrow O(G)-\delta_{n}(G)$

$\Rightarrow \mathfrak{I}_{\text {twbif }}(G) \leq O(G)-\delta_{n}(G)$

More over, $\Delta_{e}(G) \leq O(G)$ and $\delta_{e}(G) \leq \delta_{n}(G)$

$\begin{aligned} \Rightarrow \mathfrak{I}_{\text {sbif }}(G) \leq \mathfrak{\Im}_{\text {tsbif }}(G) & \leq O(G)-\Delta_{n}(G) \\ \leq O(G) & -\Delta_{e}(G)\end{aligned}$

$\Rightarrow \mathfrak{I}_{w b i f}(G) \leq \mathfrak{J}_{\text {twbif }}(G) \leq O(G)-\delta_{n}(G) \leq O(G)-$ $\delta_{e}(G)$.

Corollary 3.21: If $G$ is a bipolar intuitionistic fuzzy graph, then $\mathfrak{I}_{t s b i f}(G) \leq \mathfrak{I}_{t w b i f}(G)$.

Proof: If $x, y$ is a minimal total and strong weak dominating set respectively. If $D_{n}(i)=\Delta_{n}(G)$ and $D_{n}(j)=$ $\delta_{n}(G)$ not that $V-N(j)$ is a total weak domination.

$$
\begin{aligned}
& \mathfrak{T}_{t s b i f}(G) \leq|V-N(i)|_{\text {sbif }} \\
& \mathfrak{T}_{\text {tsbif }}(G) \leq O(G)-\Delta_{n}(G) \text { and } \mathfrak{\Im}_{t w b i f}(G) \leq \\
& |V-N(i)|_{\text {sbif }} \\
& \mathfrak{T}_{\text {twbif }}(G) \leq O(G)-\delta_{n}(G)
\end{aligned}
$$

Since $O(G)-\Delta_{n}(G) \leq O(G)-\delta_{n}(G)$ were we get $\mathfrak{I}_{\text {Esbif }}(G) \leq \mathfrak{I}_{\text {Ewbif }}(G)$

Corollary 3.22: For a bipolar intuitionistic fuzzy graph $G$

1) $O(G)-S(G) \leq \mathfrak{\Im}_{E s b i f}(G) \leq O(G)-\delta_{e}(G)$.

2) $O(G)-S(G) \leq \mathfrak{J}_{\text {Ewbif }}(G) \leq O(G)-\Delta_{e}(G)$.

Proof: Straight forward.

Note 3.23: Let $G$ is a bipolar intuitionistic fuzzy graphs such that every vertex having a same membership grade, then

1) $O(G)-S(G) \leq \mathfrak{J}_{\text {tsbif }}(G) \leq O(G)-\delta_{e}(G)$.

2) $O(G)-S(G) \leq \mathfrak{J}_{\text {twbif }}(G) \leq O(G)-\Delta_{e}(G)$.

IV. EXAMPLES FOR TOTAL STRONG DOMINATION BIPOLAR INTUITIONISTIC FUZZY GRAPH

In this section we will provide examples of total strong domination bipolar intuitionistic fuzzy graphs illustrated by figures (Fig. 4, Fig. 5 and Fig. 6), respectively.

\section{Example 4.1}

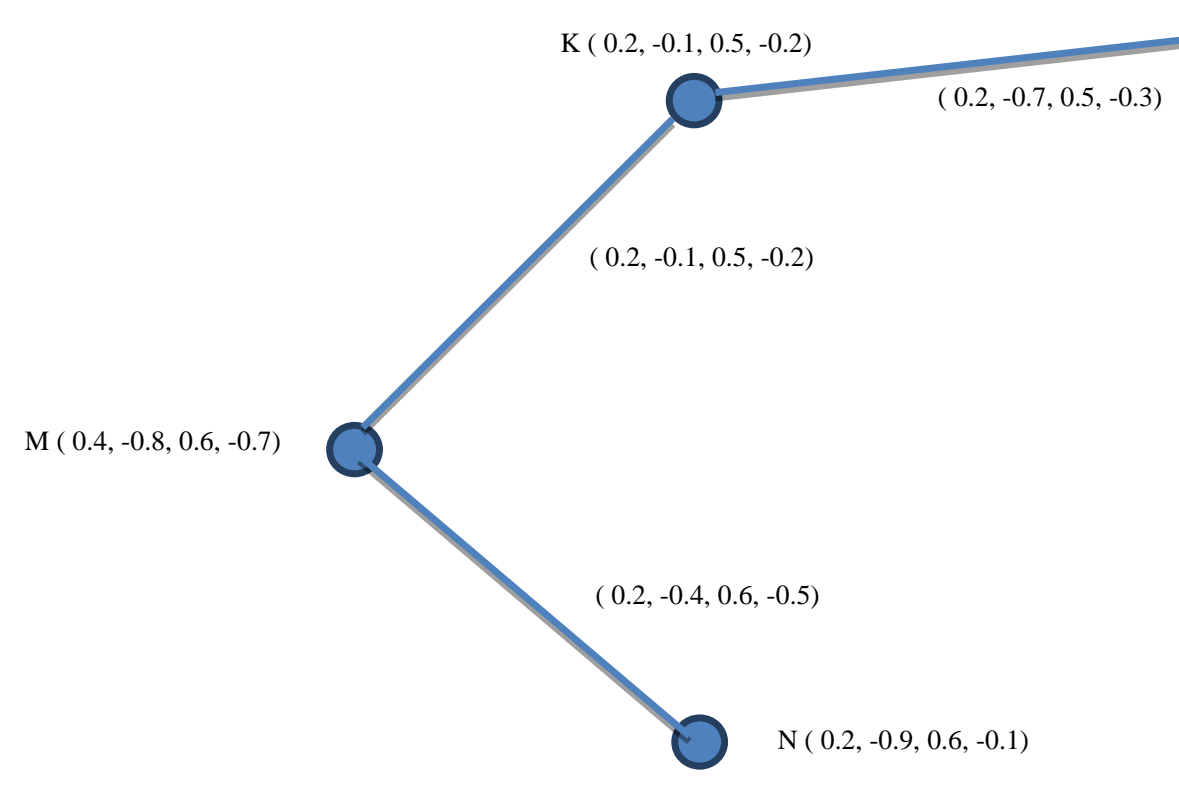

Fig. 4. Total Strong Domination Bipolar Intuitionistic Fuzzy Graphs. 
Example 4.2

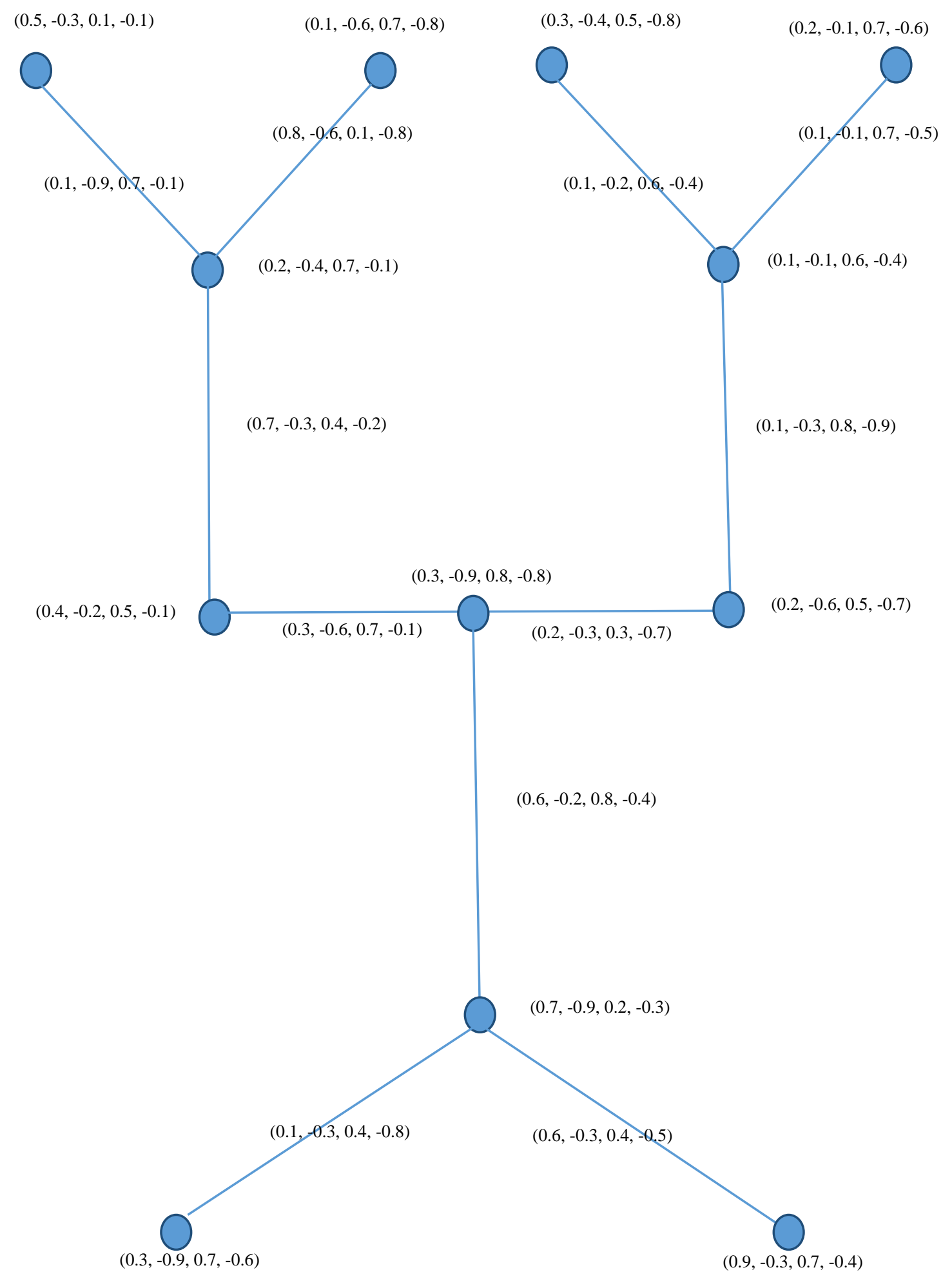

Fig. 5. Total Strong Domination Bipolar Intuitionistic Fuzzy Graphs. 
Example 4.3

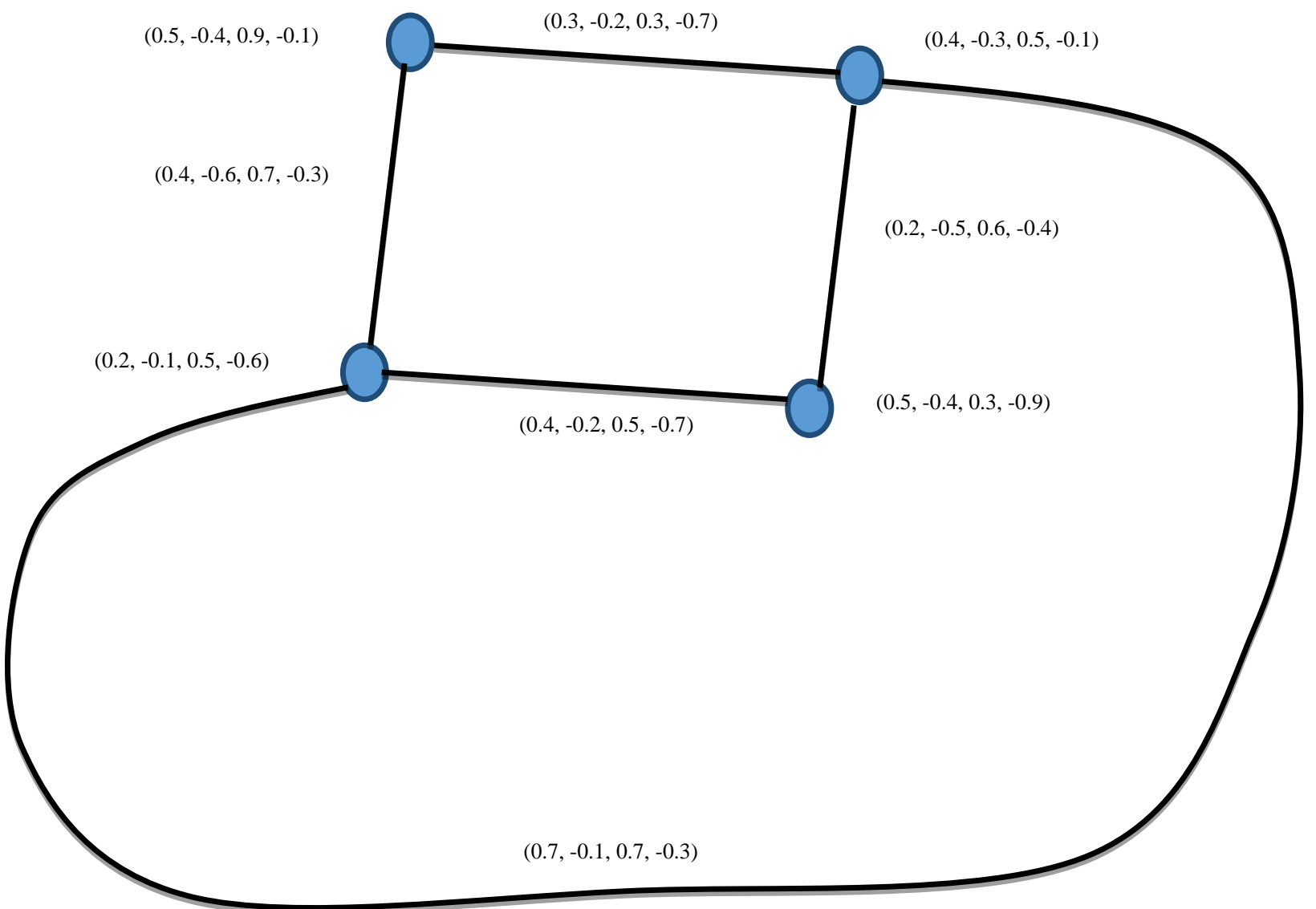

Fig. 6. Total Strong Domination Bipolar Intuitionistic Fuzzy Graphs.

\section{CONCLUSION}

Through this article, total strong (weak) domination, strong (weak) bipolar intuitionistic fuzzy graphs and some properties are discussed. In Over future paper these concepts will be generalized to hyper and tripolar fuzzy soft set, also this concept will be used in developing some topics in many papers such as [25-27].

\section{ACKNOWLEDGMENTS}

The author is very grateful to the reviewers who will give their opinion on this manuscript. He also thank to their management for their constant encouragement in scientific research.

\section{REFERENCES}

[1] L. A. Zadeh, "Fuzzy set," Information control, vol. 8, 1965, pp. 338353.

[2] A. Rosenfeld, "Fuzzy groups," Journal of Mathematical Analysis and Application, vol. 35, no. 3, 1971, pp. 512-517.

[3] A. Rosenfeld, Fuzzy Graphs, in L. A. Zadeh, Fu.k.s. shimura (E)s., "Fuzzy set and their application to cognitive and decision processes," Academic press, New York, 1975, pp. 77-95.

[4] R.T. Yeh, S.Y. Bany, Fuzzy relations and their applications to clustering analysis, in L.A.Zadeh, k.s. Fu.M. shimura (E)s. Fuzzy set and their application, Academic press, 1975, pp. 125-149.

[5] M.O.Massa'deh, Y.Al-wadi and F. Esma'el, "The fuzzy index and it's types," Far East J. Math. Sci, vol. 25, 2007, pp. 83-92.
[6] M.O.Massa'deh, "Some properties of upper fuzzy order, African journal of mathematics and computer science research," vol. 3, 2010, pp. 192194.

[7] M.O.Massa'deh, “On M-fuzzy subrings," Far East journal of mathematical sciences, vol. 62, 2011, pp. 41-49.

[8] R.M.S.Mahmood and M.O.Massa'deh, "on Groups Acting on trees of finite extensions of free groups," Mathematical Sciences letters, vol. 7 , 2018, pp. 111-116.

[9] K.T. Atanassov, "intuitionistic fuzzy sets," fuzzy sets and systems, vol. 20, 1986, pp. 87-96.

[10] M. Akram and B. Davvaz," Strong intuitionistic fuzzy graphs," Filomat, vol. 26, 2012, pp. 177-196.

[11] M.O.Massa'deh, "Structure properties of an intuitionistic anti fuzzy subgraphs," Journal of Applied Computer Science and Mathematics, vol. 7, 2013, pp. 42-44.

[12] M.O.Massa'deh, "A study on intuitionistic fuzzy and normal fuzzy Msubgroup, M-Homomorphism and Isomorphism ," International Journal of industrial mathematics, vol. 8, 2015, pp. 185-188.

[13] M.O.Massa'deh," Some contributions on intuitionistic Q-fuzzy kuIdeals," JP Journal of Algebra, Number theory and Applications, vol. 42, 2019, pp. 95-110.

[14] W.R. Zhang, "Bipolar fuzzy sets," In proceeding of Fuzz-IEEE, 1998 , pp. 835-840.

[15] M. Sunil, M.S. Sunitha and N. Anjali, "Some connectivity concepts in bipolar fuzzy graphs," Annals of pure and applied mathematics, vol. 7, 2014, pp. 98-108.

[16] M.O.Massa'deh, "On bipolar fuzzy cosets," bipolar fuzzy ideals and isomorphism of $\Gamma$-near rings, vol. 102, 2017, pp. 731-747. 
[17] M.O.Massa'deh, "A study on anti bipolar Q-fuzzy normal semi groups," Journal of Mathematical sciences and applications, vol. 6, 2018, pp. 15.

[18] W.A.Al-Zoubi , A.M.Alnaser, H.Hatamleh, Y.Alwadi and M.O.Massa'deh, "Introduction to Cartesian, tensor and lexi cographic product of bipolar interval valued fuzzy graph ," Journal of Engineering and Applied Sciences, vol. 15, 2018, pp. 581-585.

[19] K. Sankar and D. Ezhilmaran, "bipolar intuitionistic fuzzy graphs with application," International Journal of Research and Scientifical Innovation (IJRSI), vol. 3, 2016, pp. 44-52.

[20] D. Ezhilmaran and K. Sankar, " morphism of bipolar intuitionistic fuzzy graphs," Journal of Discrete Mathematical Sciences and Cryptography, vol. 18,2015 , pp. $605-621$.

[21] A.M. Alnaser, W.A. Al-Zoubi and M.O.Massa'deh, "Bipolar intuitionistic fuzzy graph and it's matrices," Applied Mathematics and information sciences, vol. 14, 2020, pp. 205-214.
[22] M. Akram and W.A. Dudak, " Regular bipolar fuzzy graphs", Neural Comput and Applic, vol.21, 2012, pp. 197-205.

[23] M. B. Sheeba and R. Pilakkat, " Strength of Fuzzy cycles", South Asian Journal of Mathematics, vol. 3, 2013, pp. 8 - 12.

[24] F. Harary, "Graph theory," Third edition, Addison-wesly, Reading , MA, 1972.

[25] A. M. Alnaser, Y. O. Kulakov, "Reliable Multipath Secure Routing In Mobile Computer Networks," Computer Engineering and Intelligent Systems, vol. 4, 2013, pp. 8-15.

[26] A. M. Alnaser, "Set-theoretic Foundations of the Modern Relational Databases: Representations of Table Algebras Operations," British Journal of Mathematics \& Computer Science, vol. 4, 2014, pp. 32863293.

[27] A. M. Alnaser, "Streaming Algorithm For Multi-path Secure Routing in Mobile Networks," IJCSI International Journal of Computer Science Issues, vol. 11, 2014, pp. 112-114. 